Chem. Phys. Lett.

\title{
Infrared Spectroscopy of the Glyoxal Radical Cation: The Charge Dependence of Internal Rotation
}

\author{
D. Leicht, ${ }^{a}$ T. C. Cheng ${ }^{\mathrm{b}}$ and M. A. Duncan ${ }^{\mathrm{b} *}$ \\ ${ }^{a}$ Lehrstuhl für Physikalische Chemie II, Ruhr-Universität Bochum, 44801 Bochum, Germany \\ ${ }^{\mathrm{b}}$ Department of Chemistry, University of Georgia, Athens, Georgia 30602, U.S.A.
}

\begin{abstract}
Glyoxal radical cations are produced in a pulsed discharge/supersonic molecular beam source. These ions are argon tagged and studied with mass-selected infrared laser photodissociation spectroscopy in the $1500-3500 \mathrm{~cm}^{-1}$ region. Five vibrational resonances are detected, three of which are assigned to two $\mathrm{CH}$ stretches and the asymmetric $\mathrm{CO}$ stretch fundamentals. Additional bands are assigned to vibrational combinations based on anharmonic frequency calculations. DFT calculations of the internal rotation coordinate, and the comparison of the predicted versus experimental IR spectra, show that the radical cation has only one stable structure, the trans conformer.
\end{abstract}




\section{Introduction}

Cis-trans isomerization plays an important role not only in organic chemistry but also in biochemistry [1]. The physical and chemical properties as well as physiological activity of a molecule strongly depend on its particular configuration. Internal rotation barriers can vary from non-existent (so called free internal rotation) to several thousand wavenumbers. In the latter case, a clear distinction between two conformers can be made. However, in the case of barrierless or low-barrier rotations one often refers to internal rotational energy levels to account for the dynamical structure in high resolution spectra. There are many examples of internal rotations in molecules, some of which show identical limiting case structures due to the permutation of identical nuclei. One example is ethane, where rotation about the $\mathrm{C}-\mathrm{C}$ bond transforms two identical structures into each other. The internal rotation leads to perturbations and splittings in the vibration-rotation-torsion energy levels [2]. These effects are present not only for the rotation of covalent bonds, but have also been shown for molecular complexes, e.g., benzene:water [3] and ammonium cation:water [4]. Internal rotation is well studied for neutral molecules and complexes, but little is known about ions. In the present work we investigate the torsional behavior upon ionization in the glyoxal cation.

Neutral glyoxal exhibits cis-trans isomerization, and both experimental and theoretical studies have investigated this molecule [5-16]. Most of the theoretical work focused on the potential energy surface along the torsional mode. One particular study investigated the ability of glyoxal to function as a molecular switch, changing conformation from trans to cis in the presence of strong electric fields [14]. Microwave and infrared spectroscopic methods have examined the ground state of glyoxal $[6,9,10,15]$, while there are also several studies of its electronic states and photochemistry [15,17-19]. The trans conformer is lower in energy than the cis, however, small amounts of the cis isomer have been observed in the gas phase [6]. The 
energy difference between the two conformers and the isomerization barriers have been determined experimentally by Parmenter and coworkers by fitting observed energies for the torsional vibration in both conformers [10]. They reported an energy difference of $1688 \mathrm{~cm}^{-1}$, a cis $\rightarrow$ trans barrier of $389 \mathrm{~cm}^{-1}$ and a trans $\rightarrow$ cis barrier of $2077 \mathrm{~cm}^{-1}$. Whereas some glyoxal derivatives (in particular methyl glyoxal) have been investigated [17,20], there is only limited information on the cis/trans isomerization of the glyoxal cation. The isomers of $\mathrm{C}_{2} \mathrm{O}_{2} \mathrm{H}_{2}{ }^{+}$, of which glyoxal is only one example, have been investigated by ab initio calculations [16]. Portwood et al. concluded from an ESR study that the glyoxal cation exists primarily in the trans conformation [21]. They speculated that the cis conformation might become stabilized by a bonding interaction between the two oxygen atoms.

In the present work, the potential energy surface of glyoxal cation is investigated via infrared laser photodissociation spectroscopy and quantum chemical calculations. Our results indicate that the glyoxal radical cation is only stable in the trans conformation.

\section{Experimental}

Glyoxal cations are produced via electrical discharge in a pulsed supersonic molecular beam, as described previously [22-26]. A the ambient vapor above a solution of $30 \%$ glyoxal in water (Sigma Aldrich) was added into a buffer gas flow containing 30\% hydrogen and 70\% argon. After the molecular beam is skimmed into the second chamber, ions are pulse-extracted into a reflectron time-of-flight mass spectrometer [27]. The ions of interest are mass selected and then intersected with the tunable output of an infrared OPO/OPA laser system at the turning point of the reflectron. Because infrared energies are not sufficient to break covalent bonds, we employ the argon tagging technique [22-26]. Spectral intensities are not corrected for the laser 
pulse energy because of the variation in laser spot size/shape and thus its overlap with the ion beam in different wavelength regions.

Density functional theory (DFT) calculations are employed to investigate the electronic structure, potential energy surface (PES), and vibrational frequencies of the glyoxal cation using the Gaussian09 program package [28]. Harmonic as well as anharmonic (VPT2) frequency calculations at the B3LYP-D3/aug-cc-pVTZ level of theory were used to assign the experimentally observed IR spectral features. Relaxed PES scans of the torsional mode of neutral glyoxal, as well as its radical cation, were obtained by changing the torsional angle stepwise and allowing all other coordinates to relax. The PES scan was conducted at the B3LYP-D3/aug-cc-pVTZ level of theory. The structures, spectra, and PES of the neutral species was investigated for comparison with previous studies to validate these methods.

\section{Results and Discussion}

The black trace (third from the top) in Figure 1 shows the experimental infrared spectrum measured for the glyoxal ${ }^{+}$Ar cation in the mass channel corresponding to the elimination of argon. It consists of a single band at $1791 \mathrm{~cm}^{-1}$ near where the carbonyl stretch vibration is expected, two bands at 2822 and $2876 \mathrm{~cm}^{-1}$ in the higher frequency region where the $\mathrm{C}-\mathrm{H}$ stretches are expected, and two weaker bands at 3076 and $3320 \mathrm{~cm}^{-1}$. The $1791 \mathrm{~cm}^{-1}$ band has a poorer signal level, most likely because the laser power is about 10x lower here than it is near $2800 \mathrm{~cm}^{-1}$. It is also conceivable that the dissociation yield is smaller here because of the lower photon energy, which is likely close to the argon binding energy. This $1791 \mathrm{~cm}^{-1}$ band can be compared to the asymmetric carbonyl stretch of neutral (trans) glyoxal at $1732 \mathrm{~cm}^{-1}$ [29]. The $\mathrm{C}-\mathrm{H}$ stretch doublet at $2822 / 2876 \mathrm{~cm}^{-1}$ can be compared to the asymmetric $\mathrm{C}-\mathrm{H}$ stretch of neutral (trans) glyoxal at $2835 \mathrm{~cm}^{-1}$ [29]. The other IR-active fundamentals of neutral glyoxal 
occur at frequencies lower than those covered by this experiment [29]. Neutral glyoxal does not have any fundamentals at frequencies higher than $2835 \mathrm{~cm}^{-1}$ where our two additional bands appear. The isolated glyoxal cation in the trans structure should have one IR-active $\mathrm{C}-\mathrm{H}$ stretch, whereas the cis structure would have two (symmetric and asymmetric motions of the two $\mathrm{CH}$ groups). The two bands near $2800 \mathrm{~cm}^{-1}$ might therefore suggest that we have the cis isomer. However, the cis glyoxal cation should also have two IR-active carbonyl stretches (symmetric and asymmetric motions of the two carbonyl groups), and we see only one band here, consistent with expectations for the trans structure. We must also remember that the cation here is tagged with argon, and that the argon binding may introduce more asymmetry in the structure and additional bands in the spectrum. Therefore, we need theory for the cation, both with and without the argon, to be able to assign this spectrum.

To further investigate this system, computational studies were conducted at the B3LYPD3/aug-cc-pVTZ level of theory on the neutral glyoxal molecule, and then on the glyoxal cation, in both the cis and trans structures for each conformer. The dispersion-corrected functional gave better results for the known cis-trans barrier in neutral glyoxal and is therefore used throughout these studies. Calculations on the cation also were done including the argon tag atom. These results are summarized in the Supporting Information. The computational results find the known stable structures for the cis and trans isomers of the neutral glyoxal, with the trans structure more stable, consistent with previous work [5-16]. The energy difference between the two conformers is calculated to be $1625 \mathrm{~cm}^{-1}$ (including only the torsional zero point energy). If all vibrational degrees of freedom are included in the ZPVE correction, the difference in energy is $1559 \mathrm{~cm}^{-1}$. The scaled harmonic frequencies for the carbonyl stretch and asymmetric $\mathrm{C}-\mathrm{H}$ stretch (1737 and $2841 \mathrm{~cm}^{-1}$, respectively) are in nice agreement with the values known for the trans neutral from experiments (1732 and $2835 \mathrm{~cm}^{-1}$ [29]), validating this level of theory. A stable trans 
configuration is also found for the glyoxal cation, with a structure qualitatively similar to that of the corresponding neutral. However, we find no stable minimum for the cis cation structure. This was confirmed by harmonic frequency calculations, which show an imaginary frequency connected to the torsional mode. The trans cation structure is analogous to that of the neutral, but its bond distances and angles are quite different, as shown in Figure 2. In particular, the $\mathrm{C}-\mathrm{C}$ bond distance is much longer in the cation, and the $\mathrm{C}-\mathrm{O}$ bond distances are shorter. The $\mathrm{H}-\mathrm{C}-\mathrm{O}$ bond angle is much greater in the cation than it is in the neutral. Argon bonding in the tagged complexes does not affect these cation structural parameters significantly.

In addition to the cis and trans structures corresponding to neutral glyoxal, the cation also has a rearranged $\mathrm{HO}(\mathrm{CH})=\mathrm{C}=\mathrm{O}^{+}$ketene-type structure lying significantly lower in energy than the trans glyoxal structure, which was reported previously [16]. Our computations find that this structure lies $19.2 \mathrm{kcal} / \mathrm{mol}$ lower than the trans glyoxal cation. Previous work on radical cations of other small molecules has also shown a similar tendency for significant rearrangement of structures $[26,30]$. However, theory predicts that this ketene-like structure has a strong $\mathrm{O}-\mathrm{H}$ stretch near 3400-3600 $\mathrm{cm}^{-1}$ and a carbonyl stretch near $2200 \mathrm{~cm}^{-1}$, and these features are inconsistent with our spectrum (see Supporting Information). Apparently, ionization of neutral glyoxal produces the ion with a similar structure, and there are significant barriers involved in rearranging to the ketene-like structure, explaining why it is not formed in our experiment.

We next examine the argon-tagged structures for the trans conformer and their vibrational patterns. We find two isomers differing in the binding position of argon on the trans cation. Isomer $\mathrm{A}$ has the argon binding in a symmetric position above the $\mathrm{C}-\mathrm{C}$ bond, whereas isomer $\mathrm{B}$ has the argon binding on a $\mathrm{CH}$ hydrogen atom. The computed energies of these isomers differ only by $0.2 \mathrm{kcal} / \mathrm{mol}$. Figure 1 shows the infrared spectra predicted for these isomers compared to the experimental spectrum. As shown, both isomers have essentially a single band at the 
position of the carbonyl stretch, although both of these have frequencies much higher than that in the experiment. In the $\mathrm{C}-\mathrm{H}$ stretching region, isomer $\mathrm{A}$ has only a single band, but isomer $\mathrm{B}$ has a doublet here, with relative intensities matching the experiment. This doublet arises from the argon binding on the $\mathrm{CH}$ hydrogen. As seen previously, when argon binds to hydrogen, that vibration shifts to lower frequency and gains intensity compared to a free-CH stretch. The 2822 $\mathrm{cm}^{-1}$ band is therefore assigned to the $\mathrm{C}-\mathrm{H}$ stretch involving the argon and the $2876 \mathrm{~cm}^{-1}$ corresponds to the other $\mathrm{C}-\mathrm{H}$ stretch. It therefore seems that the experimental spectrum agrees better with that predicted for isomer $\mathrm{B}$, with argon binding on $\mathrm{CH}$. It is also conceivable that a small amount of isomer A is present, since its main peak might overlap with the feature at 2876 $\mathrm{cm}^{-1}$. Neither computed spectrum for isomer A or B predicts the weak features at 3076 and 3320 $\mathrm{cm}^{-1}$. Because these bands lie at frequencies above the expected fundamentals of glyoxal, we suspect that they represent combinations, which would not be found in the spectra predicted by harmonic theory.

To further investigate these higher frequency bands, we calculated the anharmonic spectrum of the argon-on-CH trans conformer. We conducted anharmonic (VPT2) calculations using Gaussian09 at the B3LYP-D3/aug-cc-pVTZ level of theory. The lower trace of Figure 1 shows the spectrum predicted by this anharmonic theory, which does reproduce the two bands in the higher frequency region. A summary of the band assignments is shown together with the experimental line positions and harmonic calculations in Table 1. According to the anharmonic theory, the $3076 \mathrm{~cm}^{-1}$ band corresponds to a combination between the asymmetric $\mathrm{C}-\mathrm{O}$ stretch and the in-plane $\mathrm{CO}$ bend. The $3320 \mathrm{~cm}^{-1}$ band corresponds to the same two motions in combination with the trans in-plane $\mathrm{CH}$ bend.

We next investigated a scan of the potential energy surface in the torsional coordinate to explore why the cis conformer exists for the neutral but not the cation. Figure 3 show the 
potential energies of the neutral and radical cation glyoxal in red and blue respectively. For the neutral it is clearly seen that both conformers are stable, which was confirmed by harmonic frequency calculations. The isomerization barriers were calculated using only ZPVE correction of the torsional mode, and are $2046.5 \mathrm{~cm}^{-1}$ for the trans $\rightarrow$ cis and $422.2 \mathrm{~cm}^{-1}$ for the cis $\rightarrow$ trans barriers. These values are in reasonable agreement with the experimental values found by Parmenter and coworkers of 2077, and $389 \mathrm{~cm}^{-1}$ for the trans $\rightarrow$ cis barrier, and cis $\rightarrow$ trans barrier, respectively [10]. The highest deviation is found for the cis $\rightarrow$ trans barrier and is only about $8 \%$.

The potential energy surface of the cation (blue line in Figure 3) shows no stable cis conformer. The torsional barrier is found to be $768 \mathrm{~cm}^{-1}$ (ZPVE corrected: $685 \mathrm{~cm}^{-1}$ ). Due to the rather large barrier and considering the mass of the oxygen participating in the rotation, we do not expect to observe any tunneling effects at our resolution. However, the removal of one electron from this molecule induces a rather dramatic change in its structural and dynamical behavior.

In both the neutral and the cation, the electron-electron repulsion of the non-bonding electrons located on the oxygen atoms destabilize the cis conformation. This explains why the trans conformer is generally more stable and is in agreement with the findings of Bulat et al., who showed that the repulsive forces in the cis conformation are mainly due to through-space interactions of the $\mathrm{CO}$ groups [13]. In the neutral molecule, the presence of an electronic $\pi$ system stabilizes the cis conformer, and introduces a sufficient barrier to make this conformer locally stable (see Figure 3). Bulat et al. showed that the greatest contribution to the isomerization barrier in the neutral is such a through-bond interaction [13]. Ionization of glyoxal removes one electron from the $\mathrm{HOMO}$, which corresponds to the non-bonding lone pairs on oxygen, producing a ${ }^{2} \mathrm{~A}_{\mathrm{g}}$ ground state, but theory has shown that this orbital is close in energy 
and mixed in character with the highest $\pi$ orbital [8]. Ionization here apparently disrupts the $\pi$ system and destabilizes the cis conformer. This can be seen by the much longer $\mathrm{C}-\mathrm{C}$ bond distance in the cation (Figure 2). An additional structural change related to this is that the H-C-O bond angle expands (the C-C-O angle decreases), bringing the lone pairs of oxygen closer together in the cis configuration. Because of these combined effects, its stable minimum and barrier collapse, and the cis conformer is no longer viable.

\section{Conclusions}

Infrared photodissociation spectroscopy and DFT calculations were used to investigate the stable structures of the glyoxal cation. Infrared spectra detect the carbonyl stretch and $\mathrm{C}-\mathrm{H}$ stretch fundamentals for a single isomeric species. Anharmonic theory explains weak combination bands at higher frequency. The comparison of the experimental spectrum with that predicted by theory shows that complexation with Ar within our experiment yields only one glyoxal cation:Ar conformer, i.e. that with the $\mathrm{CH}$-bonded argon. Through a combined computational and experimental approach, we show that the glyoxal radical cation exists only in the trans conformer. A ketene-like structure computed to be more stable than the trans-glyoxal structure is not detected. The potential energy surfaces for the neutral and ionized glyoxal are quite different. The loss of $\pi$ electron density in the neutral HOMO disrupts the conjugation of the cis conformer, destabilizing the it enough so that this conformer is not stable.

\section{Acknowledgments}

This work was supported by the National Science Foundation through grant no. CHE-1464708.

D. Leicht was supported by the Cluster of Excellence RESOLV (EXC 1069) funded by the Deutsche Forschungsgemeinschaft. 


\section{References}

[1] C. Dugave, L. Demange, "Cis-trans isomerization of organic molecules and biomolecules: Implications and applications," Chem. Rev. 103 (2003) 2475.

[2] J. T. Hougen, "Perturbations in the vibration-rotation-torsion energy levels of an ethane molecule exhibiting internal rotation splittings," J. Mol. Spec. 82 (1980) 92.

[3] S. Suzuki, P. G. Green, R. E. Bumgarner, S. Dasgupta, W. A. Goddard, III, G. A. Blake, "Benzene forms hydrogen bonds with water," Science 257 (1992) 942.

[4] P. J. Kelleher, C. J. Johnson, J. A. Fournier, M. A. Johnson, A. B. McCoy, "Persistence of dual free internal rotation in $\mathrm{NH}_{4}^{+}\left(\mathrm{H}_{2} \mathrm{O}\right) \cdot \mathrm{He}_{\mathrm{n}=0-3}$ ion-molecule complexes: Expanding the case for quantum delocalization in He tagging," J. Phys. Chem. A 119 (2015) 4170.

[5] T.-K. Ha, "Ab initio calculation of cis-trans isomerization in glyoxal," J. Mol. Struc. 12 (1972) 171.

[6] J. R. Durig, C. C. Tong, Y. S. Li, "Microwave spectrum of cis-glyoxal," J. Chem. Phys. 57 (1972) 4425 .

[7] J. R. Durig, W. E. Bucy, A. R. H. Cole, "Torsional potential function of glyoxal," Can. J. Phys. $53(1975) 1832$.

[8] C. E. Dykstra, H. F. Schaefer, III, "Electronic structure of dicarbonyls: The ground state of glyoxal," J. Am. Chem. Soc. 97 (1975) 7210.

[9] C. E. Dyllick-Brenzinger, A. Bauder, "Microwave spectrum, dipole moment and barrier to internal rotation of trans-methyl glyoxal," Chem. Phys. 30 (1978) 147.

[10] K. W. Butz, D. J. Krajnovich, C. S. Parmenter, "An experimental potential energy surface for internal rotation in glyoxal," J. Chem. Phys. 93 (1990) 1557. 
[11] M. L. Senent, S. F. Herrera, F. J. Meléndez, "A comparative analysis of the QCISD(t) and the $\mathrm{CCSD}(\mathrm{T})$ potential energy surfaces for the torsional mode of glyoxal $(\mathrm{COH}-\mathrm{COH}), " \mathrm{~J}$. Mol. Struc. (THEOCHEM) 433 (1998) 161.

[12] D. P. Tew, N. C. Handy, S. Carter, "The vibrations of glyoxal, studied by 'multimode,' with a large amplitude motion, using an ab initio potential surface," Mol. Phys. 99 (2001) 393.

[13] F. Bulat, A. Toro-Labbé, "A theoretical study of the rotational isomerization of glyoxal and halogen derivatives," Chem. Phys. Lett. 354 (2002) 508.

[14] Y. Tantirungrotechai, "Effect of an external electric field and a neighboring atom on the torsional potential of glyoxal: a computational study," J. Mol. Struc. (THEOCHEM) 624 (2003) 279.

[15] R. Volkamer, P. Spietz, J. Burrows, U. Platt, "High-resolution absorption cross-section of glyoxal in the UV-Vis and IR spectral ranges," J. Photochem. Photobiol. A: Chemistry 172 (2005) 35 .

[16] D. Vijay, G. N. Sastry, "Relative energies of $\mathrm{C}_{2} \mathrm{O}_{2} \mathrm{H}_{2}$ isomers and their ionized counterparts: Possibility of bond stretch isomerism," J. Mol. Struc. (THEOCHEM) 714 (2005) 199.

[17] J. Chaiken, M. Gurnick, J. D. McDonald, "Average singlet-triplet coupling properties of biacetyl and methyl glyoxal using quantum beat spectroscopy," J. Chem. Phys. 74 (1981) 106.

[18] L. M. Dobeck, H. M. Lambert, W. Kong, P. J. Pisano, P. L. Houston, " $\mathrm{H}_{2}$ production in the 440 nm photodissociation of glyoxal," J. Phys. Chem. A 103 (1999) 10312.

[19] J. W. Hepburn, N. Sivakumar, P. L. Houston, "State-to-state photochemistry of glyoxal using a tunable VUV laser for product detection," in: Laser Techniques in the Extreme Ultraviolet, AIP Publishing, 1984, pp. 126.

[20] S. N. Wren, B. P. Gordon, N. A. Valley, L. E. McWilliams, G. L. Richmond, "Hydration, orientation, and conformation of methyl glyoxal at the air-water interface," J. Phys. Chem. A 119 (2015) 6391. 
[21] L. Portwood, C. J. Rhodes, M. C. R. Symons, "An ESR study of glyoxal radical cations," J. Chem. Soc., Chem. Comm. (1986) 1535.

[22] G. E. Douberly, A. M. Ricks, B. W. Ticknor, P. v. R. Schleyer, M. A. Duncan, "Infrared spectroscopy of the tert-butyl cation in the gas phase," J. Am. Chem. Soc. 129 (2007) 13782.

[23] G. E. Douberly, A. M. Ricks, P. v. R. Schleyer, M. A. Duncan, "Infrared spectroscopy of gas phase benzenium ions: Protonated benzene and protonated toluene from 750 to $3400 \mathrm{~cm}^{-1}$," J. Phys. Chem. A 112 (2008) 4869.

[24] G. E. Douberly, A. M. Ricks, P. v. R. Schleyer, M. A. Duncan, "Infrared spectroscopy of gas phase $\mathrm{C}_{3} \mathrm{H}_{5}^{+}$: The allyl and 2-propenyl cations," J. Chem. Phys. 128 (2009) 17.

[25] M. A. Duncan, "Infrared laser spectroscopy of mass-selected carbocations," J. Phys. Chem. A $116(2012) 11477$.

[26] J. D. Mosley, J. W. Young, M. Huang, A. B. McCoy, M. A. Duncan, "Infrared spectroscopy of the methanol cation and its methylene-oxonium isomer," J. Chem. Phys. 142 (2015) 114301.

[27] M. A. Duncan, "Reflectron time-of-flight mass spectrometer for laser photodissociation," Rev. Sci. Instrum. 63 (1992) 2177.

[28] M. J. Frisch, G. W. Trucks, H. B. Schlegel, G. E. Scuseria, M. A. Robb, J. R. Cheeseman, G. Scalmani, V. Barone, B. Mennucci, G. A. Petersson, H. Nakatsuji, M. Caricato, X. Li, H. P. Hratchian, A.F. Izmaylov, J. Bloino, G. Zheng, J. L. Sonnenberg, M. Hada, M. Ehara, K. Toyota, R. Fukuda, J. Hasegawa, M. Ishida, T. Nakajima, Y. Honda, O. Kitao, H. Nakai, T. Vreven, J.A. Montgomery Jr., J. E. Peralta, F. Ogliaro, M. Bearpark, J. J. Heyd, E. Brothers, K. N. Kudin, V.N. Staroverov, R. Kobayashi, J. Normand, K. Raghavachari, A. Rendell, J. C. Burant, S.S. Iyengar, J. Tomasi, M. Cossi, N. Rega, J. M. Millam, M. Klene, J. E. Knox, J. B. Cross, V. Bakken, C. Adamo, J. Jaramillo, R. Gomperts, R. E. Stratmann, O. Yazyev, A. J. Austin, R. Cammi, C. Pomelli, J. W. Ochterski, R. L. Martin, K. Morokuma, V. G. Zakrzewski, G. A. Voth, 
P. Salvador, J. J. Dannenberg, S. Dapprich, A. D. Daniels, Ö. Farkas, J. B. Foresman, J. V. Ortiz, J. Cioslowski, D. J. Fox, Gaussian, Inc., Wallington CT, 2009.

[29] T. Shimanouchi, Molecular Vibrational Frequencies, NIST Chemistry WebBook, NIST Standard Reference Database Number 69, Linstrom, P. J.; Mallard, W. G., eds., National Institute of Standards and Technology, Gaithersburg, MD, 20899 (http://webbook.nist.gov).

[30] J. L. Holmes, C. Aubrey, P. M. Mayer, Assigning Structures to Ions in Mass Spectrometry, CRC Press, Boca Raton, 2007. 
Table 1. Vibrational frequencies $\left(\mathrm{cm}^{-1}\right)$ observed by the experiment compared to those predicted by harmonic and anharmonic theory.

\begin{tabular}{llll}
\hline Experiment & Harmonic $^{\mathrm{a}, \mathrm{b}}$ & Anharmonic $^{\mathrm{c}}$ & Assignment \\
\hline 1791 & 1948 & 1949 & asym. C-O stretch \\
2822 & 2821 & 2723 & $\mathrm{C}-\mathrm{H} \cdots$ Ar stretch \\
2876 & 2877 & C-H stretch \\
3076 & NA & 3005 & asym. C-O stretch/CH bend comb. \\
3320 & NA & 3310 & asym. C-O stretch/CH bend/CO bend comb. \\
& & \\
& &
\end{tabular}




\section{Figure Captions}

Figure 1. Experimental spectrum in black alongside scaled (0.968) harmonic frequencies for the two stable glyoxal:argon complexes as well as the anharmonic (VPT2) spectrum (blue, lower trace) for the $1 \mathrm{H}$-bound conformer.

Figure 2. The bond distance and bond angle changes that take place in glyoxal upon ionization.

Figure 3. Potential energy scan along torsional coordinate for neutral and radical cation glyoxal in red and blue, respectively. 


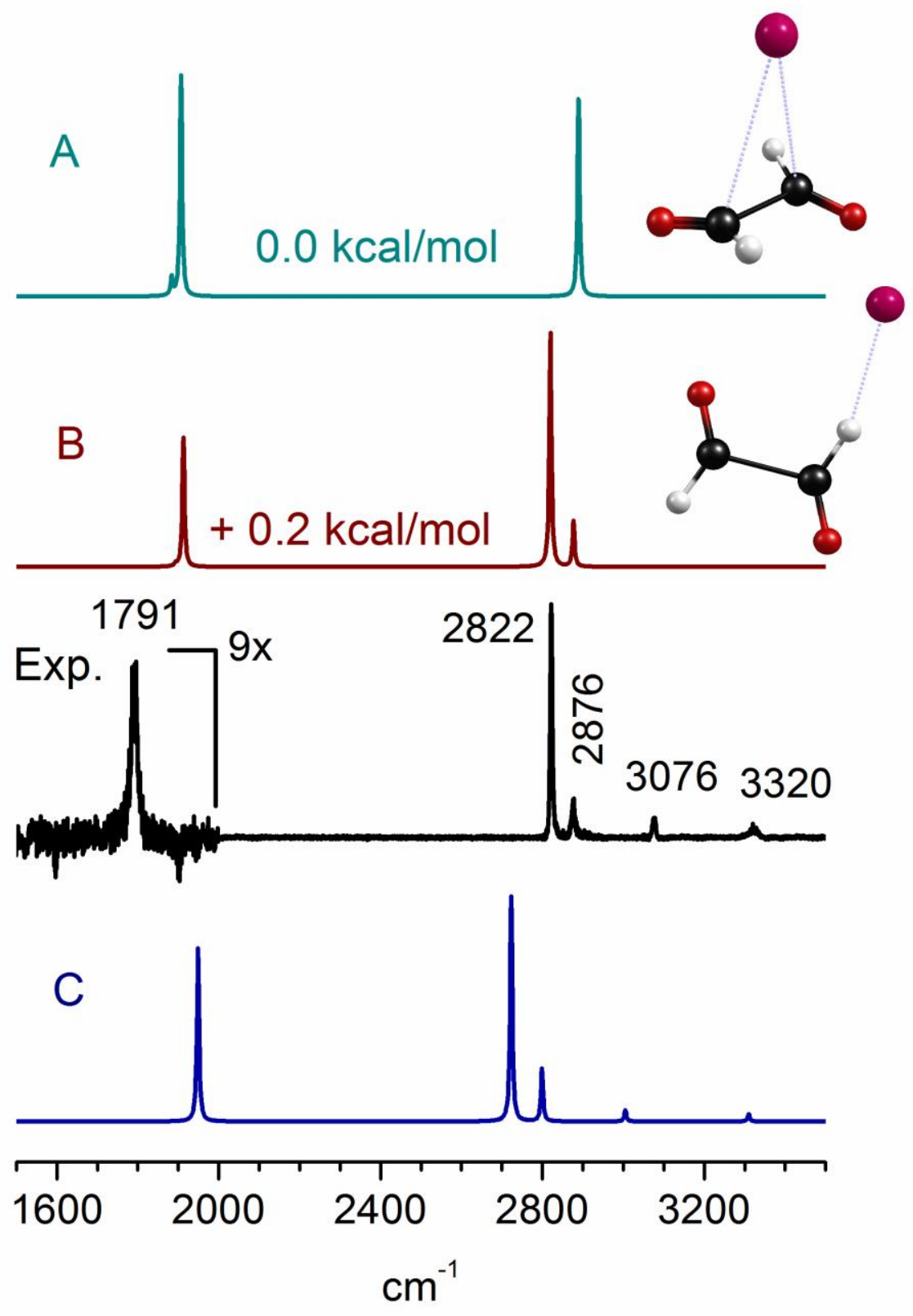

Figure 1. 


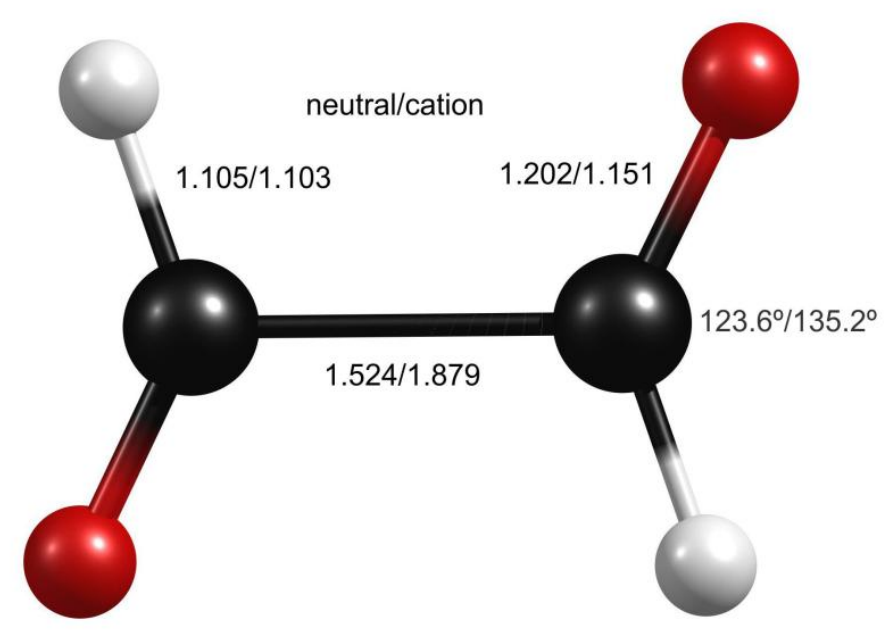

Figure 2. 


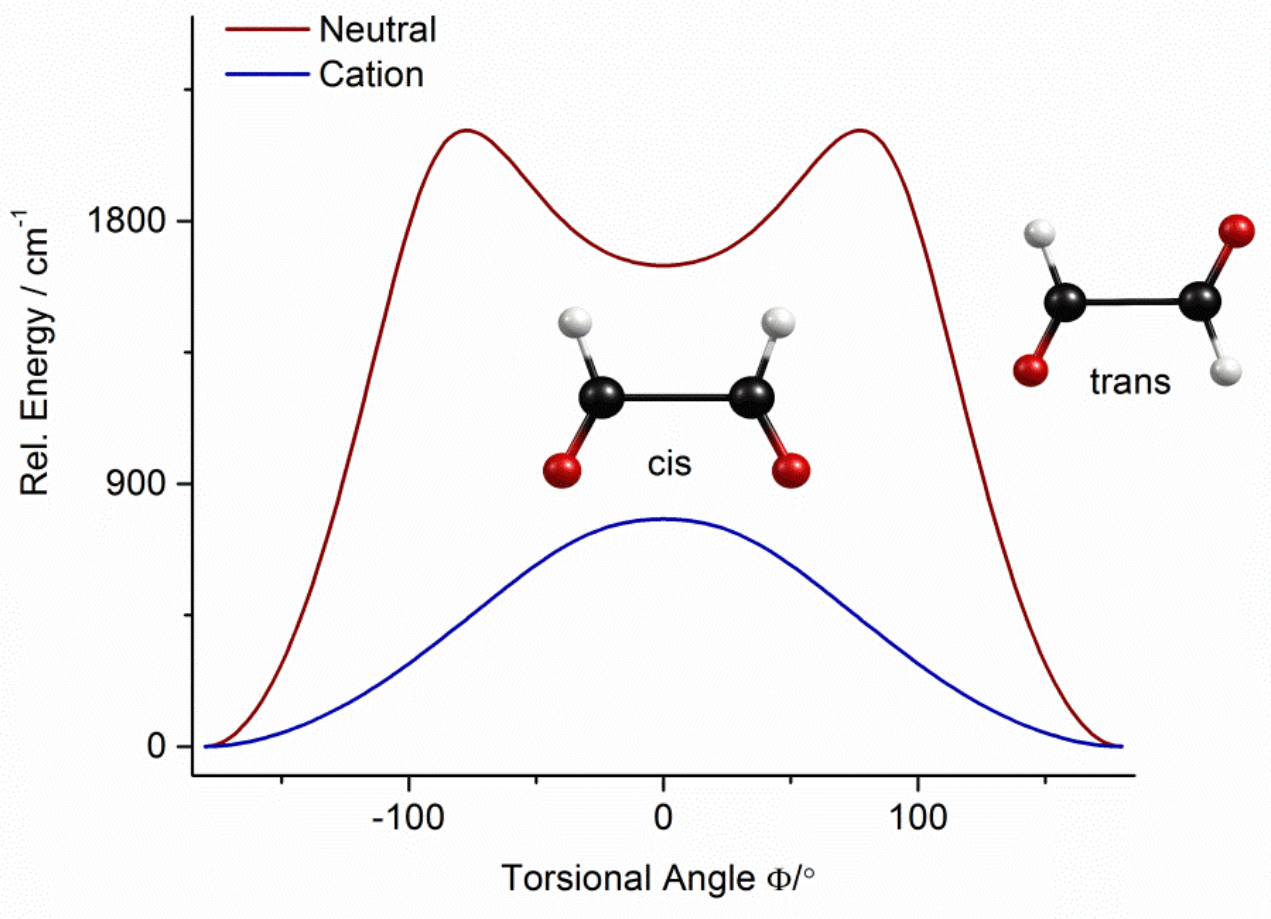

Figure 3. 
Table of Contents Graphic:

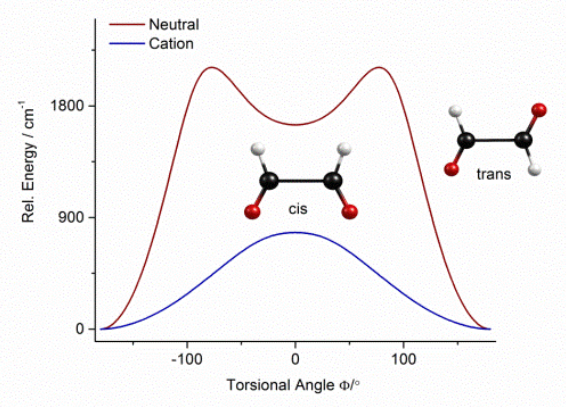

